\title{
Periodontal Health among Type 2 Diabetes Patients Treated with Different Dental Restorations
}

\author{
Mohammad Albakry ${ }^{*}{ }^{\circledR}$, Michael Vincent Swain ${ }^{2}{ }^{\circledR}$, Abdo Abdul-Razzaq $^{3}{ }^{(0)}$, \\ Falah Alshammary ${ }^{4}\left(\mathbb{0}\right.$, , Waleed Asiri $^{3}$ (i) \\ ${ }^{1}$ Restorative Dental Sciences, Faculty of Dentistry, University of Najran, Najran, KSA \\ ${ }^{2}$ Biomaterials Science Research Unit, School of Aerospace, Mechanical and Mechatronic Engineering, Biomechanics Engineering, \\ The University of Sydney, Sydney, New South Wales, Australia \\ ${ }^{3}$ Preventive Dental Sciences, Faculty of Dentistry, University of Najran, Najran, KSA \\ ${ }^{4}$ Preventive Dental Sciences, Faculty of Dentistry, University of Hail, Hail, KSA \\ Email: ^albakrym@hotmail.com, michael.swain@sydney.edu.au,dr_alrazki2004@yahoo.com, \\ falah_R_M_SH@hotmail.com,w1111s@hotmail.com
}

How to cite this paper: Albakry, M., Swain, M.V., Abdul-Razzaq, A., Alshammary, F. and Asiri, W. (2021) Periodontal Health among Type 2 Diabetes Patients Treated with Different Dental Restorations. Open Journal of Stomatology, 11, 278-296.

https://doi.org/10.4236/ojst.2021.118025

Received: June 28, 2021

Accepted: August 2, 2021

Published: August 5, 2021

Copyright (c) 2021 by author(s) and Scientific Research Publishing Inc. This work is licensed under the Creative Commons Attribution International License (CC BY 4.0).

http://creativecommons.org/licenses/by/4.0/

\begin{abstract}
Background: Diabetes increases periodontal disease risk and its complications, which are worsened with age or faulty restorations. Aim: Evaluate periodontal health among patients with diabetes treated with different dental restorations. Materials and Methods: This comparative cross-sectional study was conducted at the faculty of dentistry, Najran University, KSA, from March 2018 to February 2020. It included 260 diabetics, treated with different dental restorations, divided into four groups, each comprising 65 patients aged 45 - 60 years: Group 1 (G1), no restorations (control group); Group 2 (G2), Class II amalgam restorations; Group 3 (G3), Class II composite resin restorations; and Group 4 (G4), three-unit posterior metal ceramic prostheses. The Ages of all restorations ranged from 8 to 10 years. All groups were assessed for the Plaque Index (PI) and Gingival Index (GI), Overhangs Rate (OR), Alveolar Bone Loss (ABL) (the latter two using panoramic X-ray). HbAlc and duration of participants were also assessed and compared. Results: The mean $\mathrm{HbAlc}$ results of the groups ranged from $8.1 \%$ to $9.5 \%$, and their mean diabetes durations ranged from 7.2 to 12 years. All the groups showed worse periodontal scores, ranging from $2.45-2.95$ for PI and $2.25-2.8$ for GI. G2 had higher scores than G1 and G3 $(p<0.001)$. G4 had the highest two recorded scores ( $p$ $<0.001)$. G2 had a higher rate of overhangs than G3 $(p<0.037)$. The mean ABL was 44\% (SD 6.4) for G1, 56.0\% (SD 6.7) for G2, 46.5\% (SD 5.8) for G3, and $74.5 \%$ (SD 6.4) for G4. The lowest values were recorded in G1 and G3 ( $p$ $=0.987)$. The highest value was observed in G4, which was significantly high-
\end{abstract}


er than other groups $(p<0.001)$. Conclusion: The worse periodontal parameters among subjects treated with either three-unit metal ceramic or Class II amalgam restorations indicated severe periodontal destruction. Untreated subjects and subjects treated with Class II composite resin had relatively better periodontal status.

\section{Keywords}

Diabetes Mellitus, Alveolar Bone Loss, Plaque Index, Dental Restoration

\section{Introduction}

Diabetes mellitus, which is typically referred to as diabetes, is a progressive metabolic condition associated with abnormally high concentrations of blood glucose, also known as hyperglycemia [1]. It is a chronic non-communicable disease that affects the way the human body metabolizes glucose. Type 2 diabetes was formerly referred to as non-insulin-dependent diabetes or adult diabetes [2]. Globally, the prevalence of diabetes has risen rapidly, particularly in developing countries [3]. In fact, poorly controlled type 2 diabetes can result in serious damage to various human organs, such as the heart, kidneys, eyes, and oral tissues [4]. This disease also increases the risk of periodontal disease and its complications by approximately 3 to 4 times [5]. Furthermore, the severity of periodontal complications is significantly dictated by maintaining normal glucose levels. Likewise, periodontal disease can have a serious influence on the metabolic control of diabetes [6]. Although the two chronic diseases are linked by an established bidirectional relationship [7], the detailed mechanism by which they negatively affect each other remains ambiguous. However, researchers have speculated that hyperglycemia results in the development and accumulation of advanced glycation end-products [8] [9]. These end products interact with their receptors (RAGEs) in the periodontal complication site, thus inducing proinflammatory cytokines that promote periodontal inflammation and degradation of alveolar bone [10].

Periodontal illness is a plaque-induced chronic inflammatory condition affecting tooth tissues and their surroundings due to bacterial accumulations [11]. It involves a wide variety of inflammatory disorders that have an effect on the structures that support the teeth, such as gingiva, bone, and periodontal ligaments [12]. Poor oral hygiene is a very well-known etiological factor for periodontal disease [13]. The lack of appropriate and adequate tooth brushing and other essential oral hygiene measures can result in poor oral hygiene status. This condition promotes the accumulation of harmful bacteria and the buildup of pathogenic dental plaque biofilm on the hard and soft tissues of the oral cavity [14]. The repercussion of such a condition is linked to a stage of inflammatory alteration in the oral tissues. Such periodontal alteration is quantified or diagnosed by examining the patient's basic clinical oral signs and assessing certain 
periodontal indices. Periodontal indices, such as Plaque Index (PI) and Gingival Index (GI), are dental expressions of clinical observation, usually presented in numerical values that describe the incidence, prevalence, or severity status of a periodontal condition [15]. They are among the most important periodontal parameters used in determining periodontal diseases.

Alveolar bone loss is the resorption or recession of bone that surrounds and supports the teeth [16]. Alveolar bone loss is a common and widespread disease, affecting $90 \%$ of the global population [17]. It is most commonly caused by not replacing lost teeth, especially multiple teeth [18]. Chronic periodontitis, and its associated periodontal complications, such as a high level of dental plaque and severe gingival inflammation, is another common cause of alveolar bone loss [19]. In such cases, bacteria gradually attack the periodontal ligaments that connect the tooth to the alveolar bone, causing the destruction of these ligaments and subsequent alveolar bone loss [20].

Dental restorations play a major role in the preservation of periodontal health [21]. The long-term performance and high success rate of Class II restorative treatments are significantly influenced by healthy gingiva and its surrounding structures. For example, the anatomical location of Class II restorations and the nature of the restorative material render Class II restorations either difficult to maintain and/or clean. Class II cavity preparation encompasses the removal of a significant portion of the decayed occlusal surface as well as a portion of the proximal surface. Thus, after restoration is placed, the patient might find it difficult to keep the restoration surface and interface between the restoration and the cavity margin hygienic and free of plaque, especially in the interproximal area. Such an unhygienic proximal area is a suitable site for further bacterial growth and its resultant inflammation progression. Moreover, the restoration's overhang encourages further plaque formation among Class II restorations.

Overhang is known as the extension of a dental restoration beyond the confines of a prepared cavity [22]. The overhang presents an obstacle for proper oral hygiene, because it minimizes necessary access to interproximal cleaning devices and can cause severe inflammation of adjacent soft tissues. A prior study reported that an overhanging dental restoration is a significant etiological factor responsible for promoting plaque accumulation, thus increasing periodontal disease development and the risk of secondary caries [23]. Nevertheless, it should be made clear that the dimensions of the overhang are indispensable in this regard, as an overhanging margin $\leq 0.2 \mathrm{~mm}$ should be harmless to periodontal health [23] [24] [25].

In fact, the relationship between periodontal health and dental restorations has been investigated considerably for decades. Many research investigations and reviews have shown that among patients treated with different dental restorations, especially faulty restorations, worse periodontal parameters, resulting from chronic periodontitis, are common findings [26]-[31]. In fact, the effect is even worse when periodontal disease occurs alongside other chronic diseases, 
such as diabetes mellitus. It is well established that poor glycemic control is associated with diabetes-related dental conditions, such as an increased incidence of periodontitis, periodontal attachment loss, alveolar bone loss, tooth loosening, and, consequently, tooth loss [32] [33] [34].

In our rigorous review of the literature, we were unable to locate any study appraising the impact of different dental restorative treatments on the periodontal health of patients with type 2 diabetes mellitus. Hence, in the current study, we aimed primarily at determining the amount of alveolar bone loss among patients with type 2 diabetes treated with different dental restorations. We also assessed the oral health indices, PI, and GI of these patients. Furthermore, we evaluated and compared the prevalence of Class II overhanging restorations among subjects treated with Class II amalgam and composite resin restorations.

\section{Materials and Methods}

This research project was approved by the Research and Ethics Committee of the Faculty of Dentistry, Najran University, Kingdom of Saudi Arabia (research project approval No. 001/18, January 1, 2018). In the current study, clinical examinations and/or evaluations that involved humans were conducted in accordance with the ethical standards of the institutional and/or national research committee, and the Helsinki Declaration, as amended by the $64^{\text {th }}$ WMA General Assembly, Fortaleza, Brazil, October 2013, and its later amendments or comparable ethical standards. In addition, before commencing the research, we obtained signed consent forms from all examined patients who participated in the current study.

\subsection{Sample Size and Sampling}

To calculate the sample size, we adopted the formula proposed by Crano and Brewer [35] for calculating sample size in medical research, as follows:

$$
n=N n^{*} / N+n^{*}
$$

where $n$ is the required sample size, $N$ is the population size (total number of participants, 844), and $n^{*}$ is the first estimated sample. The first estimated sample $\left(n^{*}\right)$ was determined using the following formula:

$$
n^{*}=P(1-P) /(\mathrm{SE})^{2},
$$

where $P$ is the estimated proportion of participants, which was assumed as 0.5 for obtaining the maximum sample size, and SE is the standard error, assumed as 0.05 . Therefore, the first estimated sample is $n^{*}=100$; consequently, the sample size $(n)$ is 89.4 . It should be noted that any further increment in the population size would have limited effect on the results [35].

\subsection{Inclusion and Exclusion Criteria}

The inclusion and exclusion criteria for the selection of the participants adopted in the current study were as follows: 


\section{Inclusion Criteria}

The inclusion criteria included: 1) Medically diagnosed type 2 diabetes mellitus $\geq 2$ years; 2) 45 - 60 years of age; 3) HbA1c levels $\geq 6.5 \%$; 4) A minimum of 15 teeth remaining; and 5) Class II amalgam restorations, Class II composite resin restorations, posterior metal ceramic restorations; age 8 - 10 years.

\section{Exclusion Criteria}

The exclusion criteria included: 1) Self-reported medical chronic conditions, such as HIV, cardiovascular complications, hepatic disorder, renal disorders, or epilepsy; 2) A history of previous antibiotic use or steroid therapy for the past three weeks; 3) Immunosuppressive chemotherapy; 4) Periodontal treatment for the past six months; 5) Edentulous patients; and 6) Crowding teeth or occlusal trauma.

\section{Study Population}

In this observational comparative cross-sectional study, we selected 260 patients with type 2 diabetes patients among a sample of 844 patients with diabetes who presented to the specialized dental clinics of the Faculty of Dentistry, Najran University, Kingdom of Saudi Arabia. Patients were divided into four groups according to their restorative treatment types. Each group comprised 65 patients, aged 45 - 60 years: Group 1 (G1) had no restorative treatment (control group), Group 2 (G2) had Class II amalgam restorations, Group 3 (G3) had Class II composite resin restorations, and Group 4 (G4) had three-unit posterior metal ceramic prostheses. Because all participants were divided based on the type of restoration, we excluded participants with more than one type of restoration. The service life of all restorations ranged from 8 to 10 years; we calculated the mean ages of restorations for all groups. It should be noted that female subjects were not included in the current study, because the dentistry program of the current educational institution (University of Najran) is specified for male students only. Therefore, this study sample included men only.

\subsection{Clinical Examinations}

All participants underwent clinical oral examination, including the status of periodontal tissues. Because this was an observational comparative cross-sectional study, the clinical examination of subjects was conducted using their current dental status. Moreover, no dental treatment was provided to the subjects before the clinical examination. The clinical screening examination included evaluating the state of periodontal tissues by assessing the amount of dental plaque, PI, and gingival condition and qualitative alterations of the gingiva and GI using the two scoring systems proposed by: 1) Silness and Löe [36] for PI; and 2) Löe and Silness [37] for GI. For the evaluation of PI, four sides per tooth (buccal, lingual, mesial, and distal) were examined for each participant (except for the third molars), using William's periodontal probe. For the assessment of GI, certain teeth were chosen for this purpose: 16, 12, 24, 32, 36, and 44 .

Two periodontal investigators collected the periodontal parameters, PI, and GI. Values obtained were compared, and the overall kappa score for intraexa- 
miner reliability was calculated.

\subsection{Radiographic Procedure}

Panoramic X-ray radiography was used to measure alveolar bone loss and locate sites of overhanging restorations. The panoramic X-ray unit (Planmeca Promax, Dent-R100, RPX253665, Helsinki, Finland) was used to produce the necessary images. The desired resolution of each radiographic procedure was selected, followed by adjusting the height of the X-ray based on patient height. The patient's chin was placed in a chin cup, and the occlusal plane was set to horizontal. The patient was directed to grasp the handles to tighten the head support. The laser position was adjusted to correspond with the illustration on the touch screen. We performed final and fine adjustments to the volume location when needed. We used a computer-assisted system to digitize and analyze all panoramic radiographs for linear measurements.

All panoramic radiographs and alveolar bone loss measurements for all participants were taken during regular daily dental practice visits between March 2018 and February 2020.

\section{Panoramic Radiographs}

A total of 65 complete sets of panoramic radiographs were obtained from $65 \mathrm{pa}-$ tients with diabetes, from each of the following groups.

Group 1 (untreated diabetes patients, control group)

We examined each panoramic radiograph of the 65 patients with diabetes and selected one area in the posterior segment that demonstrated the highest amount of alveolar bone. We decided to perform this selection because the comparison of the alveolar bone loss of the four groups must be considered according to the most affected sites. Therefore, 65 sites were selected to measure the mean alveolar bone loss.

Group 2 (diabetes patients, treated with Class II amalgam restorations)

We selected 49 participants, each of whom had one Class II amalgam restoration, and 16 participants, each of whom had two Class II amalgam restorations. The total number of Class II amalgam restoration was 81 . We selected 81 interproximal sites, toward the restored proximal site, to measure the mean alveolar bone loss.

Group 3 (diabetes patients, treated with Class II composite restorations)

We chose 55 participants, each of whom had one Class II composite resin restoration, and 10 participants, each of whom had two Class II composite resin restorations. The total number of Class II composite restorations was 75 . Therefore, 75 interproximal areas, toward the restored proximal side, were selected for measuring mean alveolar bone loss.

Group 4 (diabetes patients, treated with three-unit metal ceramic prostheses)

We chose 65 participants. Each patient had one metal ceramic restoration (three units, posterior segment); the total number of metal ceramic prostheses was 65. Because each metal ceramic prostheses had two abutments (four prox- 
imal sites), 260 sites were selected to measure the mean alveolar bone loss.

Among the treated posterior teeth, we considered the mean alveolar bone loss along with overhanging restorations of Class II restorations irrespective of their class (premolars or molars), site (upper or lower), or side (mesial or distal).

The criteria for acceptability of the panoramic radiographs were as follows: 1) clear visibility of the anatomical features, such as the Cementoenamel Junction (CEJ), Alveolar Bone Crest (ABC), and tooth Apices (AP); 2) CEJs were not compromised by the presence of a restoration, prostheses, traversing images, or defective radiographic image; and 3) both proximal sites (mesial and distal) were measurable. At the end of the selection process for the set of images, we discarded radiographic images that did not meet the criteria. We used a computer screen to amplify and visualize images.

The distance between the CEJ and crest of the alveolar bone, and between the crest of the alveolar bone and tooth apex, was used to identify the alveolar bone with bone loss (Figure 1). Alveolar bone loss was defined as a distance $>2 \mathrm{~mm}$ between the CEJ and the ABC. The alveolar bone loss was presented as the percentage of bone loss [38]. The percentage of bone loss percentage was calculated using the following formula [38]:

$$
\frac{(\mathrm{CEJ}-\mathrm{ABC})-2 \mathrm{~mm}}{(\mathrm{CEJ}-\mathrm{AP})-2 \mathrm{~mm}} \times 100
$$

All clinical data were collected by two periodontal investigators. Collected data were grouped onto Excel spreadsheets to record the percentage of alveolar bone level, root length, and bone loss in millimeters. The assessments of alveolar bone loss produced by the two investigators were compared, and the overall

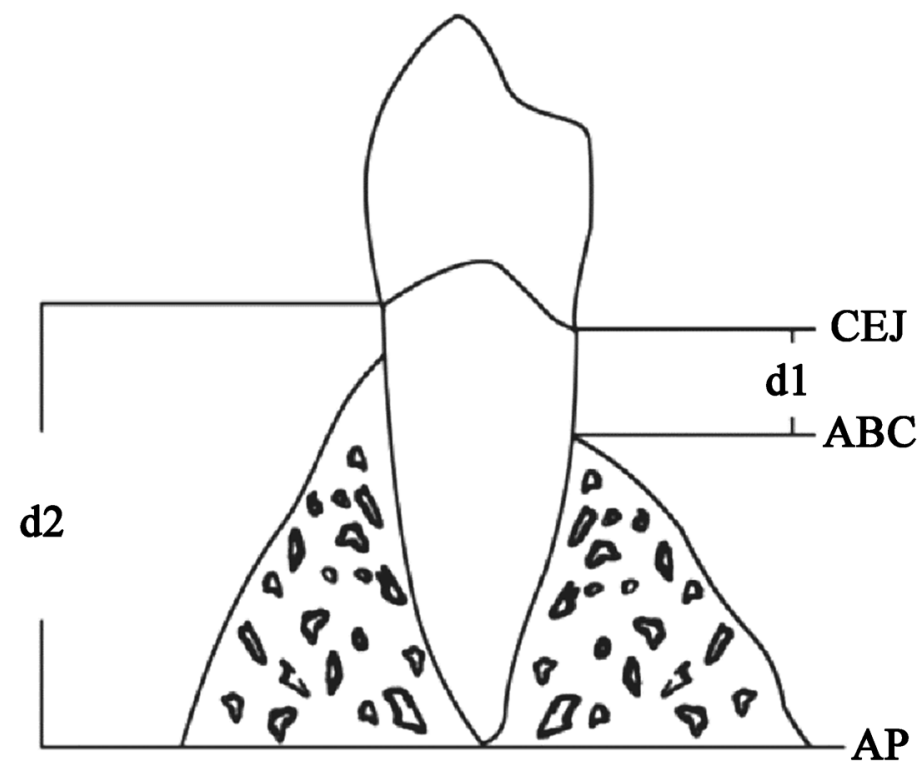

Figure 1. Illustration showing the anatomical features of a tooth used to measure the mean alveolar bone loss. CEJ indicates cementoenamel junction; ABC: alveolar bone crest; AP: apex. $\mathrm{d} 1$ is the distance form alveolar bone crest to the cementoenamel junction, and $\mathrm{d} 2$ is the distance from tooth apex to the cementoenamel junction. 
kappa score for intraexaminer reliability was calculated.

\subsection{Statistical Analysis}

We statistically evaluated the data using IBM SPSS version 25 . The variables were expressed as mean \pm SD and analyzed by means Scheffé test and one-way analysis of variance (ANOVA). A $p$ value $<0.05$ was considered statistically significant, and a $p$ value $<0.0001$ was deemed extremely significant.

\section{Results}

Socio-demographic characteristics of all subjects are provided in Table 1. These features include: number of subjects, mean age, nationality, gender, level of education, and marital status. Some of these features are known to affect periodontal health, such as gender and level of education. However, they have not been discussed in the current study, since the scope of this study is to determine the influence of specific risk factors, such as age, glycemic status, type of dental restoration. Factors that are known to be associated with periodontal change, which have not been considered in the current study, have been regarded as limitations of the study.

Table 1. Sociodemographic characteristics of the participants of the examined groups.

\begin{tabular}{|c|c|c|c|c|c|}
\hline - & - & G1 & G2 & G3 & G4 \\
\hline Number of Diabetic patients & - & 65 & 65 & 65 & 65 \\
\hline Mean age (years) (SD) & - & $51.2(5.4)$ & $52(5)$ & $51.8(4.3)$ & $57(4.9)$ \\
\hline \multirow{7}{*}{$\begin{array}{c}\text { Nationality } \\
\text { No. (\%) }\end{array}$} & Saudi & $44(67.6)$ & $37(56.9)$ & $50(76.9)$ & $40(61.5)$ \\
\hline & Yemini & $9(13.8)$ & $16(24.6)$ & $4(6.1)$ & $5(7.7)$ \\
\hline & Egyptian & $7(10.7)$ & $8(12.3)$ & $3(4.6)$ & $3(4.6)$ \\
\hline & Sudanese & $5(7.7)$ & $3(4.6)$ & $2(3)$ & $10(15.3)$ \\
\hline & Indian & 0 & $1(1.5)$ & $2(3)$ & $2(3)$ \\
\hline & Bengali & 0 & 0 & $3(4.6)$ & $4(6.1)$ \\
\hline & Bakistani & 0 & 0 & $1(1.5)$ & $1(1.5)$ \\
\hline \multirow{2}{*}{$\begin{array}{l}\text { Gender } \\
\text { No. (\%) }\end{array}$} & Male & $65(100)$ & $65(100)$ & $65(100)$ & $65(100)$ \\
\hline & Female & 0 & 0 & 0 & 0 \\
\hline \multirow{4}{*}{$\begin{array}{c}\text { Marital status } \\
\text { No. (\%) }\end{array}$} & Single & 0 & 0 & 0 & 0 \\
\hline & Married & $59(90.7)$ & $55(84.6)$ & $62(11)$ & $56(86.1)$ \\
\hline & Widowed & 0 & 0 & $3(4.6)$ & $8(12.3)$ \\
\hline & Divorced & $6(9.2)$ & $10(15.3)$ & 0 & $1(1.5)$ \\
\hline \multirow{4}{*}{$\begin{array}{c}\text { Education } \\
\text { No. }(\%)\end{array}$} & Elementary & $31(47.6)$ & $25(38.4)$ & $33(50.7)$ & $36(55.3)$ \\
\hline & Secondary & $29(44.6)$ & $30(46.1)$ & $22(33.8)$ & $20(30.7)$ \\
\hline & Graduate & $5(7.7)$ & $8(12.3)$ & $10(15.3)$ & $9(13.8)$ \\
\hline & Postgraduate & 0 & $2(3)$ & 0 & 0 \\
\hline
\end{tabular}


The mean ages of the participants in groups G1 - G4 were 51.2 (SD 5.4), 52 (SD 5), 51.8 (SD 4.3), and 57 (SD 4.9) years, respectively (Table 2). There was no significant difference in the mean ages of the first three groups, G1 - G3 ( $p=$ 0.392). The participants in G4 were significantly older than those in the other three groups, G1 - G3 ( $p=0.03)$.

Table 2 lists the glycemic status and diabetes durations of all examined groups. HbAlc ( \pm SD) mean levels of the participants in G1 - G4 were 8.5 (SD 0.38), 9.4 (SD 0.9), 8.1 (SD 1.14), and 9.3 (SD 0.95), respectively. The mean durations of diabetes mellitus $( \pm \mathrm{SD})$ for the same groups were 7.2 (SD 0.33), 10.8 (SD 0.36), 9.4 (SD 1.2), and $12(0.9)$, respectively. The ANOVA test showed that the diabetes durations of all groups (G1 - G4) differed significantly ( $p<0.001)$; G4 had significantly longer durations than the other groups $(p<0.001)$.

The mean service life of the restorations in the patients in G2 - G4 were 8.14 (SD 0.56), 9.17 (SD 0.63), and 9.3 (SD 0.65) years, respectively (Table 3 ). There was no significant difference in the age of restorations between G2 and G4 ( $p=$ $0.04)$, and the restorations in both groups were significantly older than the restorations of $\mathrm{G} 3(p=0.025)$.

Table 3 lists the PI and GI scores. The mean values of these indices for G1-G4 were 2.45 and 2.25 (SD 0.10, 0.21), 2.8 and 2.5 (SD 0.22, 0.27), 2.5 and 2.25 (SD $0.28,0.23$ ), and 2.95 and 2.8 (SD $0.10,0.24$ ), respectively. No statistically significant difference in the two values were observed between G1 and G3 $(p=0.420)$. G2 had significantly higher values than G1 and G3 did $(p<0.001)$. G4 had the statistically highest two values of all groups $(p<0.001)$. A comparison of the assessment made by the two investigators produced an overall kappa score of 0.93 for intraexaminer reliability, suggesting a good agreement between the two investigators.

Table 2. Mean age of patients with diabetes (y), HbAlc, and duration of diabetes (y).

\begin{tabular}{ccccc}
\hline Groups & No. of subjects & $\begin{array}{c}\text { Mean age of subjects } \\
\text { (years) (SD) }\end{array}$ & $\begin{array}{c}\text { Mean HbAlc } \\
(\text { SD) }\end{array}$ & $\begin{array}{c}\text { Mean duration of } \\
\text { diabetes (years) (SD) }\end{array}$ \\
\hline G1 & 65 & $51.2(5.4)$ & $8.5(0.38)$ & $7.2(0.33)$ \\
G2 & 65 & $52(5)$ & $9.4(0.9)$ & $10.8(0.36)$ \\
G3 & 65 & $51.8(4.3)$ & $8.1(1.14)$ & $9.4(1.2)$ \\
G4 & 65 & $57(4.9)$ & $9.3(0.95)$ & $12(0.9)$
\end{tabular}

Table 3. Mean service life of restorations, Mean plaque index, and Mean gingival index scores. SD = standard deviation.

\begin{tabular}{cccc}
\hline Groups & $\begin{array}{c}\text { Mean service life of } \\
\text { restorations (SD) years }\end{array}$ & $\begin{array}{c}\text { Mean plaque } \\
\text { index (SD) }\end{array}$ & $\begin{array}{c}\text { Mean gingival } \\
\text { index (SD) }\end{array}$ \\
\hline G1 & - & $2.45(0.10)$ & $2.25(0.21)$ \\
G2 & $8.14(0.65)$ & $2.8(0.22)$ & $2.5(0.27)$ \\
G3 & $9.17(0.63)$ & $2.5(0.28)$ & $2.25(0.23)$ \\
G4 & $9.3(0.65)$ & $2.95(0.10)$ & $2.8(0.24)$ \\
\hline
\end{tabular}


With regard to the overhang of the Class II restorations, chi-square test showed that diabetes patients treated with Class II amalgam restorations (G2) had a significantly higher rate of overhanging restorations $(58 \%)$ than those treated with Class II composite resin restorations (G3; 41.3\%; $p=0.037$ ). Table 4 lists the findings related to overhanging restorations.

Figure 2 charts the results of alveolar bone loss and the standard deviation for all examined groups. The mean percentages of alveolar bone loss $( \pm \mathrm{SD})$ recorded in G1 - G4 were $44 \%( \pm 4.61 \%), 56.5 \%( \pm 1.82 \%), 46.5 \%( \pm 3.82 \%)$, and $74.5 \%$ $( \pm 4.44 \%)$, respectively. ANOVA revealed that patients in G4 (diabetes patients with metal ceramic prosthesis) demonstrated the highest mean percentage of alveolar bone loss among the four groups $(p<0.001)$. The smallest amount of alveolar bone loss was observed in G1 (untreated, control group) and G3 (diabetes patients with Class II composite resin restorations), with no significant difference between them $(p=0.987)$. G2 patients (diabetes patients treated with Class II amalgam restorations) showed significantly higher alveolar bone loss than those in G1 and G3 $(p<0.001)$. Comparison of the assessment of the alveolar bone loss made by the two investigators produced an overall kappa score of 0.85 for intraexaminer reliability.

Figures 3-6 show representative alveolar bone loss images for all examined groups. Figure 3 shows a panoramic image for an un-treated diabetic patient, where the least amount of alveolar bone loss among groups is indicated by arrows in the interproximal area between 15 and 16, and the interproximal area

Table 4. Overhangs among patients with diabetes treated with Class II amalgam restorations (G2) and Class II composite resin restorations (G3).

\begin{tabular}{ccccc}
\hline Groups & $\begin{array}{c}\text { Total No. of } \\
\text { subjects }\end{array}$ & $\begin{array}{c}\text { Total No of } \\
\text { restorations }\end{array}$ & $\begin{array}{c}\text { Total No. of } \\
\text { overhanging } \\
\text { restorations }\end{array}$ & $\begin{array}{c}\text { Prevalence of } \\
\text { overhanging } \\
\text { restorations } \%\end{array}$ \\
\hline G2 & 65 & 81 & 47 & $58 \%$ \\
G3 & 65 & 71 & 31 & $41.3 \%$ \\
\hline
\end{tabular}

\section{Mean Alveolar \\ Bone Loss \%}

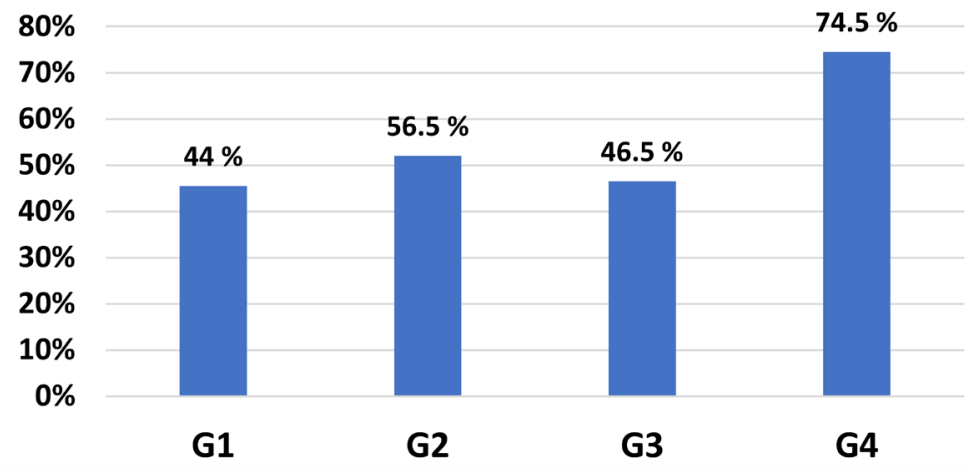

Figure 2. Mean percentage of alveolar bone loss among the four examined groups. 


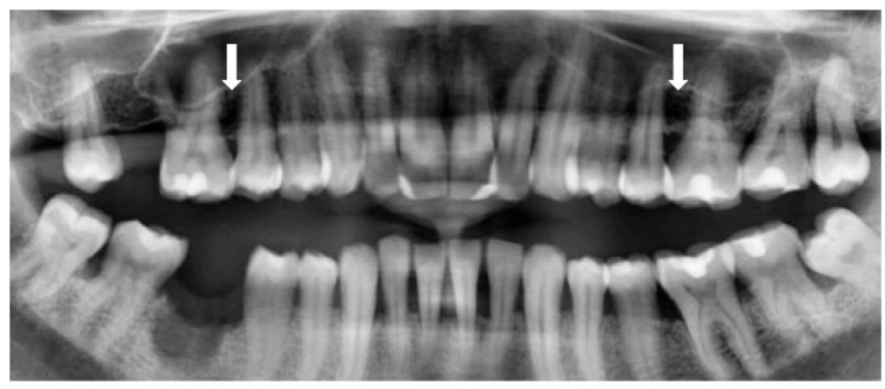

Figure 3. Diabetes patient with no restorative treatment. The area of bone loss is indicated by arrows: 1) Interproximal area between 15 and 16;2) Interproximal area between 25 and 26.

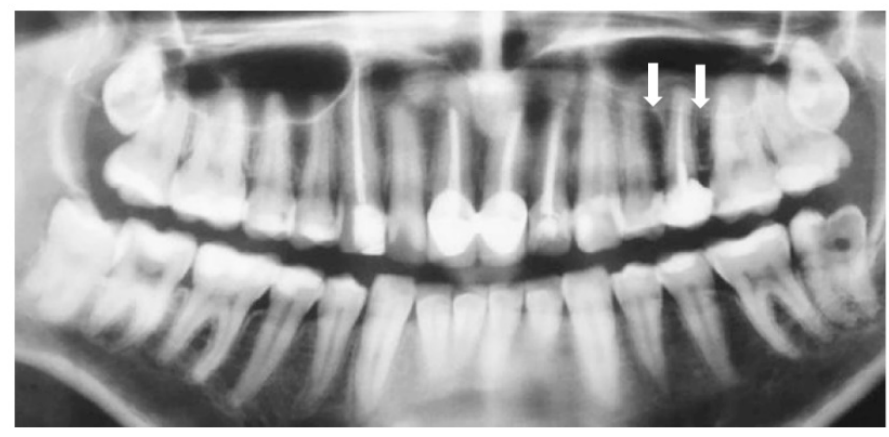

Figure 4. Diabetes patient treated with dental amalgam (Class II). The area of bone loss is indicated by arrows in the interproximal areas between: 1) 24 and 25; and 2) 25 and 26.

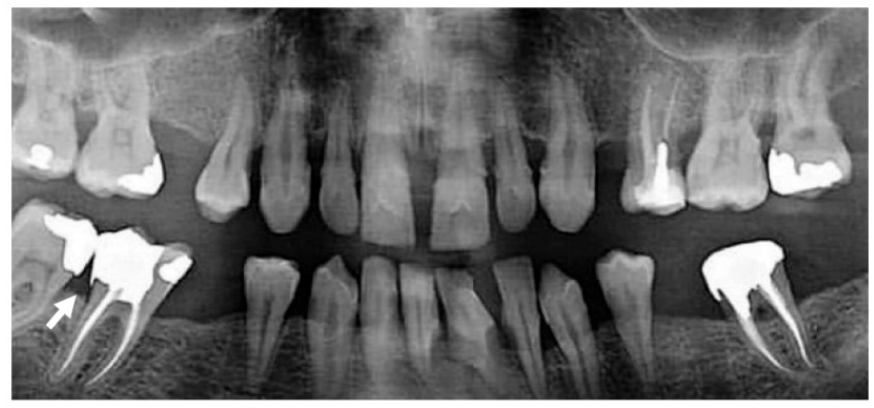

Figure 5. Diabetes patient treated with composite resin (Class II). The area of bone loss is indicated by arrow of the interproximal area between 46 and 47 .

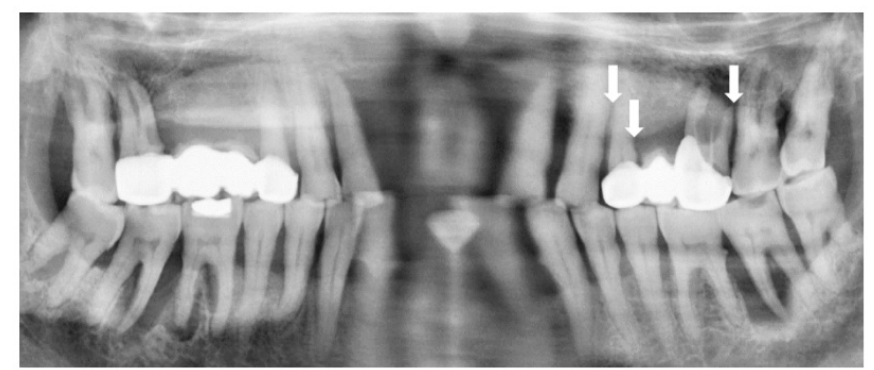

Figure 6. Diabetes patient treated with metal ceramic restorations. The area of bone loss for the three-unit metal ceramic prostheses is indicated by arrows: 1) Interproximal area between 23 and 24; 2) Interproximal area between 24 and 25; (3) Interproximal area between 26 and 27. 
between 25 and 26. Similar amount of alveolar bone loss is given in Figure 5, for a diabetic patient treated with Class II composite resin. Higher amount of alveolar bone loss that exceeded $50 \%$ of total root length is given in Figure 4 (diabetic patient treated with Class II amalgam restorations), and in Figure 6 (diabetic patient treated with 3-unit posterior metal ceramic restorations).

\section{Discussion}

There are two essential risk factors that should be considered when evaluating the periodontal health status of patients with diabetes: 1) Glycemic status, represented by HbAlc; and 2) Duration of diabetes. The HbAlc levels of the examined groups were considerably high in the current study. Although hyperglycemia is significantly associated with worse periodontal parameters, it should be noted that a longer duration of an uncontrolled glycemic status can be more destructive to periodontal tissues than a merely high $\mathrm{HbAlc}$ level. The worst periodontal parameters of the examined groups of the current study were observed among participants with longer diabetes duration, i.e., $>10$ years, G2 - G4. The relatively better periodontal parameters among the participants of G1 and G3 were observed with shorter diabetes duration, $<10$ years. Similar findings that are related to the effect of glycemia duration were reported previously. A study by Al-Shammari et al. [39] reported that patients with longer duration of diabetes had worse periodontal parameters when compared with patients with a shorter duration. Another comparative study by Abduljabbar et al. [40] found worse periodontal parameters among diabetic and prediabetic patients compared with non-diabetics. These investigators stated that the severity of periodontal inflammation is significantly dictated by the duration of hyperglycemia. These outcomes along with the current findings might lead to a speculation that the periodontal scores in patients with diabetes is significantly dictated by the duration of hyperglycemia. However, it must be emphasized that this influence could occur with considerable variation due to the inclusion of other possible risk factors, such as poor oral hygiene, faulty dental restorations, tobacco smoking, hypertension, and older age.

The mean values of the alveolar bone loss in the current study exceeded 50\% among patients with diabetes treated with: 1) Class II amalgam restorations (G2); and 2) Three-unit metal ceramic prostheses (G4). Upon clinical examination, most of the patients with diabetes in the two groups showed poor oral hygiene; the mean PI and GI index scores were the highest among all examined groups. These rather high oral health scores (worse periodontal parameters), accompanied by poor oral health status, reaffirm that severe chronic periodontitis was predominant among the subjects of the two groups and hence must be regarded as a significant predisposing factor for their worse periodontal parameters.

In this study, the worst periodontal parameters were noticed among the G4 patients. This can be attributed to several risk factors: 1) Excessive forces applied on abutments of the metal ceramic prostheses; 2) Diabetes mellitus; 3) Poor oral 
hygiene; 4) Duration of insertion of metal ceramic prostheses; and 5) Age of the patients (they were statistically the oldest among the four groups). In fact, this extremely high value was anticipated because more risk factors were involved with this treatment, compared with the risk factors of the other treatments. Combined detrimental factors can result in severe chronic periodontitis, to which most periodontal complications are attributed.

The PI and GI scores of G4 were in accordance with those of a previous study by Al-Sinaidi and Preethanath [26]. This study reported that metal ceramic prostheses were accompanied by high PI and GI scores and also greater probing pocket depths, as compared with non-abutment teeth. In addition, the authors observed that the subgingival margins of the crowns resulted in higher PI and GI scores and deeper probing than crowns with supragingival margins.

The second highest scores of the periodontal parameters were reported among G2 subjects. There were fewer risk factors resulting in these outcomes than those involved in the metal ceramic prostheses. These were: 1) Diabetes mellitus; 2) Poor oral hygiene; and 3) Overhanging restorations. The prevalence of overhangs among Class II amalgam restorations was considerably high (58\%). In addition to being an etiological factor that promotes periodontal inflammation, secondary caries, onsite and adjacent alveolar bone loss [23], an overhanging restoration can act synergistically with other factors, which eventually results in high alveolar bone loss, as seen in this group. In previously published data, the prevalence of overhanging Class II amalgam restorations varied considerably (16.5\% - 76\%) [27] [41]. This variation could be attributed to the sizes of samples examined, the size of the overhanging margin, the different experience and/or skills among dental practitioners, and various techniques adopted in treatment methodology.

As compared with G2 and G4, the smaller amount of alveolar bone loss observed in G1 and G3 (<50\%) was accompanied by lower mean PI and GI scores, which is an indication of moderate chronic periodontitis among these patients. Although the least amount of alveolar bone loss was recorded in this study by the control group (G1;44\%), it was not significantly lower than that recorded by the subjects of G3 (46.5\%). Three essential risk factors were considered to be crucial for the interpretation of the loss of alveolar bone recorded by the subjects of G3 (overhanging composite restorations, diabetes mellitus, and poor oral hygiene), whereas only two risk factors for G1 (diabetes mellitus and poor oral hygiene). Despite the difference in the number of risk factors of the two groups, there was no significant difference in the amount of alveolar bone loss or in the mean PI and GI scores, which is a little surprising.

Periodontal health and dental restorations are significantly related. There are numerous data available on the effects of different dental restorations on periodontal parameters and their association with periodontal health. The most appraised periodontal parameters are: 1) PI; 2) GI; 3) Probing depth; and 4) Clinical attachment loss. The scores of various periodontal indices among patients 
treated with different dental restorations have been reported. Al-Sanjray et al [30] found that the PI and GI of Class II amalgam restorations $(1.16,1.5)$, were comparable with those of Class II composite restorations (1.1, 1.38). Another study by Ababnaeh et al. [28] investigated the effect of different dental restoration classes, such as II, III, and V, and different dental restoration types, such as crowns and bridge abutment, on patients' periodontal health. They stated that Class II restorations had the highest PI (0.99) and also the highest probing depth scores $(2.77 \mathrm{~mm})$. On the other hand, different outcomes were reported by Al-Fawaz et al. [29], who evaluated the PI and GI for a number of posterior teeth restored with Class II amalgam and Class II composite restorations. This study reported higher PI and GI scores of Class II composite resin $(1.73,1.58)$ as compared with those of Class II amalgam restorations $(1.57,1.24)$. A recent study by Al-Abdaly et al. [31] compared the effect of Class II amalgam restorations with that of metal ceramic crowns on patients' periodontal health. Class II amalgam restorations had higher PI and GI scores than those of metal ceramic crowns.

The outcomes of the periodontal parameters reported in the present study of all groups, namely, the PI and GI scores, were higher than all those of previous reports found in the literature. One explanation for such differences might be the different clinical conditions. Previous periodontal research investigations [28] [31] that reported lower PI and GI examined patients with no systemic disease, whereas the present study evaluated the periodontal indices of individuals with diabetes. It should be noted that different outcomes might be obtained when comparing results under dissimilar clinical conditions.

Numerous observational studies, including a large number of prospective studies, have supported a correlation between diabetes, the severity of periodontal disease, attachment loss, and alveolar bone loss. In 1991, Emrich et al. [42] observed the severity of periodontal disease and its associated complications in the Pima Indian population, which has the most prevailing rate of type 2 diabetes in the world, over 2 years. These researchers found that type 2 diabetes increased the probability of developing periodontal disease threefold, irrespective of age, sex, and oral hygiene status. Taylor et al. [43] stated that patients with diabetes who have impaired glycemic control had a high risk of alveolar bone loss and greater progression than patients without diabetes. In 2014, the effect of glycemic control on periodontal health status and alveolar bone loss was assessed by Javed et al. [44]. They found that patients with poor glycemic control had a higher severity of periodontal parameters and alveolar bone loss. In a review by Wu et al. [45], the authors reported that alveolar bone loss, resulting from severe periodontitis, had an inflammatory effect on both osteoclasts and osteoblasts. In addition, diabetes was found not only to affect bone loss and coupled bone growth but also to adversely affect innate and adaptive immune responses [46]. These overall findings suggest that diabetes is a prime predisposing factor for periodontal complications and its consequences. 
Various previous studies have investigated the effect of specific factors on periodontal health, such as restorative material type, quality of restoration, overhanging restoration, aging, and supra- or subgingival restoration margins. However, no studies were identified that appraised the effect of diabetes mellitus on periodontal disease in the presence of dental restorations and subsequent alveolar bone loss. Hence, it was difficult to compare the current findings with previous research reports.

There are certain limitations of the current study that might have biased the results. All subjects of the current study were males. It is well documented that the likelihood of periodontal complications rises as a result of hormonal changes in females [47]. Although the incidence of periodontal disease has been shown to be higher in males [48], there remains a need to evaluate whether there is a difference in the severity of periodontal disease and its subsequent alveolar bone loss between females and males. A well-known risk factor for periodontal health is age. To obtain a comparable age group, we did not assess patients older than 60 years in the current study.

With respect to chronic diseases (systemic factors), the current study lacks accurate data on diabetes onset, and also the way diabetes was controlled (treatment). Moreover, we did not assess the obesity status of the subjects. Obesity is a well-known risk factor for periodontal disease and can be associated with poor periodontal parameters [49]. The patient's smoking statusus is another limitation of the present study. Smoking is a major risk factor for many medical problems, and periodontal health is not an exception [50]. Some limitations related to dental restorations should not be overlooked, such as the smoothness of Class II restorations and the size of proximal overhangs. Factors associated with metal ceramic restorations include subgingival or supragingival margins and/or space underneath the pontic, which might have affected the progression of disease. Further research that considers different aspects of these limitations is needed.

\section{Conclusion}

Within the limitations of the current study, it can be concluded that there were worse periodontal parameters among all examined diabetes groups, which manifested as high alveolar bone loss (>50\%) and high PI and GI scores among subjects treated with either Class II amalgam restorations or three-unit metal ceramic prostheses. These dental treatments appeared to be associated with extensive periodontal destructions, caused by severe chronic periodontitis. The untreated subjects and subjects treated with Class II composite resin restorations had a smaller amount of alveolar bone loss $(<50 \%)$ and lower PI and GI scores, which indicated relatively better periodontal status.

\section{Data Availability Statement}

The datasets used or analyzed during the present investigation are available from the corresponding author on request. 


\section{Funding}

The authors declare that they have not received any specific funding or other financial support for the present investigation.

\section{Acknowledgements}

The authors are very grateful to the Faculty of Dentistry, University of Najran, KSA, and would like to acknowledge the following people for their clinical assistance as well as for providing the necessary illustrations and images; Dr. Mishary Alwadiey and Dr. Abu Baker Basheer for the statistical analysis advice.

\section{Authors' Contribution}

All authors had substantial contributions to conception, investigation, clinical examination, design, and data analysis. Dr. Albakry wrote the manuscript and revised the first version. Professor Michael Swain revised and edited the manuscript critically for important intellectual content. All authors have revised and approved the final version to be published.

\section{Conflicts of Interest}

The authors declare no conflicts of interest regarding the publication of this paper.

\section{References}

[1] Naujokat, H., Kunzendorf, B. and Wiltfang, J. (2016) Dental Implants and Diabetes Mellitus-A Systematic Review. International Journal of Implant Dentistry, 2, 5. https://doi.org/10.1186/s40729-016-0038-2

[2] American Diabetes Association (2009) Diagnosis and Classification of Diabetes Mellitus. Diabetes Care, 32, 62-67. https://doi.org/10.2337/dc09-S062

[3] Ramachandran, A., Snehalatha, C., Shetty, A.S., et al. (2012) Trends in Prevalence of Diabetes in Asian Countries. World Journal of Diabetes, 3, 110-117.

https://doi.org/10.4239/wjd.v3.i6.110

[4] Nazir, M.A., AlGhamdi, L., AlKadi, M., et al. (2018) The Burden of Diabetes, Its Oral Complications and Their Prevention and Management. Open Access Macedonian Journal of Medical Sciences, 6, 1545-1553. https://doi.org/10.3889/oamjms.2018.294

[5] Chen, T.L., Xu, E.L., Lu, H.J., et al. (2012) The Influence of Diabetes Enhanced Inflammation on Cell Apoptosis and Periodontitis. Advances in Bioscience and Biotechnology, 3, 712-719. https://doi.org/10.4236/abb.2012.326092

[6] Liu, R., Bal, H.S., Desta, T., et al. (2006) Diabetes Enhances Periodontal Bone Loss through Enhanced Resorption and Diminished Bone Formation. Journal of Dental Research, 85, 510-514. https://doi.org/10.1177/154405910608500606

[7] Preshaw, P.M. and Bissett, S.M. (2019) Periodontitis and Diabetes. British Dental Journal, 227, 577-584. https://doi.org/10.1038/s41415-019-0794-5

[8] Ramasamy, R., Vannucci, S.J., Yan, S.S., et al. (2005) Advanced Glycation end Products and RAGE: A Common Thread in Aging, Diabetes, Neurodegeneration, and Inflammation. Glycobiology, 15, 16-28. https://doi.org/10.1093/glycob/cwi053 
[9] Chang, P.C., Chien, L.Y., Yeo, J.F., et al. (2013) Progression of Periodontal Destruction and the Roles of Advanced Glycation End Products in Experimental Diabetes. Journal of Periodontology, 84, 379-388. https://doi.org/10.1902/jop.2012.120076

[10] Manouchehr-Pour, M., Spagnuolo, P.J., Rodman, H.M., et al. (1981) Comparison of Neutrophil Chemotactic Response in Diabetic Patients with Mild and Severe Periodontal Disease. Journal of Periodontology, 52, 410-415. https://doi.org/10.1902/jop.1981.52.8.410

[11] Kim, J. and Amar, S. (2006) Periodontal Disease and Systemic Conditions: A Bidirectional Relationship. Odontology, 94, 10-21. https://doi.org/10.1007/s10266-006-0060-6

[12] Kinane, D.F., Stathopoulou, P.G. and Papapanou, P.N. (2017) Periodontal Diseases. Nature Reviews Disease Primers, 3, 17038. https://doi.org/10.1038/nrdp.2017.38

[13] Lockhart, P.B., Brennan, M.T., Thornhill, M., et al. (2009) Poor Oral Hygiene as a Risk Factor for Infective Endocarditis-Related Bacteremia. Journal of the American Dental Association, 140, 1238-1244. https://doi.org/10.14219/jada.archive.2009.0046

[14] Berger, D., Rakhamimova, A., Pollack, A. and Loewy, Z. (2018) Oral Biofilms: Development, Control, and Analysis. High Throughput, 7, 24.

https://doi.org/10.20944/preprints201808.0174.v1

[15] Dhingra, K. and Vandana, K.L. (2011) Indices for Measuring Periodontitis: A Literature Review. International Dental Journal, 61, 76-84.

https://doi.org/10.1111/j.1875-595X.2011.00018.X

[16] Jonasson, G., Skoglund, I. and Rythén, M. (2018) The Rise and Fall of the Alveolar Process: Dependency of Teeth and Metabolic Aspects. Archives of Oral Biology, 96, 195-200. https://doi.org/10.1016/j.archoralbio.2018.09.016

[17] Armitage, G.C. (2004) Periodontal Diagnoses and Classification of Periodontal Diseases. Periodontology 2000, 34, 9-21. https://doi.org/10.1046/j.0906-6713.2002.003421.x

[18] Atwood, D.A. (1971) Reduction of Residual Ridges: A Major Oral Disease Entity. The Journal of Prosthetic Dentistry, 26, 266-279. https://doi.org/10.1016/0022-3913(71)90069-2

[19] Wang, C.J. and McCauley, L.K. (2016) Osteoporosis and Periodontitis. Current Osteoporosis Reports, 14, 284-291. https://doi.org/10.1007/s11914-016-0330-3

[20] Li, X., Kolltveit, K.M., Tronstad, L., et al. (2000) Systemic Diseases Caused by Oral Infection. Clinical Microbiology Reviews, 13, 547-558. https://doi.org/10.1128/CMR.13.4.547

[21] Brunsvold, M.A. and Lane, J.J. (1990) The Prevalence of Overhanging Dental Restorations and Their Relationship to Periodontal Disease. Journal of Clinical Periodontology, 17, 67-72. https://doi.org/10.1111/j.1600-051X.1990.tb01064.x

[22] Padbury, A., Eber, R. and Wang, H.L. (2003) Interactions between the Gingiva and the Margin of Restorations. Journal of Clinical Periodontology, 30, 379-385. https://doi.org/10.1080/00016357.2017.1303848

[23] Jeffcoat, M.K. and Howell, T.H. (1980) Alveolar Bone Destruction Due to Overhanging Amalgam in Periodontal Disease. Journal of Periodontology, 51, 599-602. https://doi.org/10.1902/jop.1980.51.10.599

[24] Parsell, D.E., Streckfus, C.F., Stewart, B.M., et al. (1998) The Effect of Amalgam Overhangs on Alveolar Bone Height as a Function of Patient Age and Overhang Width. Operative Dentistry, 23, 94-99. 
[25] Ercoli, C., Tarnow, D., Poggio, C.E., et al. (2021) The Relationships between ToothSupported Fixed Dental Prostheses and Restorations and the Periodontium. Journal of Prosthodontics, 30, 305-317. https://doi.org/10.1111/jopr.13292

[26] Al-Sinaidi, A. and Preethanath, R.S. (2014) The Effect of Fixed Partial Dentures on Periodontal Status of Abutment Teeth. Saudi Journal of Dental Research, 5, 104-108. https://doi.org/10.1016/j.ksujds.2013.11.001

[27] Sirajuddin, S., Narasappa, K.M., Gundapaneni, V., et al. (2015) Iatrogenic Damage to Periodontium by Restorative Treatment Procedures: An Overview. The Open Dentistry Journal, 9, 217-222. https://doi.org/10.2174/1874210601509010217

[28] Ababnaeh, K.T., Al-Omari, M. and Alawneh, T.N. (2018) The Effect of Dental Restoration Type and Material on Periodontal Health. Oral Health \& Preventive Dentistry, 9, 395-403.

[29] Al-Fawaz, Y., Alofi, R. and Diab, H. (2017) Comparative Study between the Effect of Class II Amalgam and Composite Restorations in Posterior Teeth on Periodontal Tissues Health. Egyptian Dental Journal, 63, 2571-2577. https://doi.org/10.21608/edj.2017.76208

[30] Al-Sanjary, S.A. and Gasgoos, S.S. (2017) Effect of Class II Amalgam and Composite Restorations on Periodontal Health of Posterior Teeth: An in Vivo Study. Journal of Oral and Dental Research, 4, 82-92. https://doi.org/10.12816/0038706

[31] Al-Abdaly, M.M.A.A., Khawshal, A.A.Q., Alqisi, A.Y., et al. (2018) Clinical and Radiographic Evaluation of Marginal Bone Loss and Periodontal Parameters after Various Dental Reconstruction Procedures. International Journal of Clinical Medicine, 9, 39-48. https://doi.org/10.1080/00016357.2017.1303848

[32] Cervino, G., Terranova, A., Briguglio, F., et al. (2019) Diabetes: Oral Health Related Quality of Life and Oral Alterations. BioMed Research International, 2019, Article ID: 5907195. https://doi.org/10.1155/2019/5907195

[33] Khader, Y.S., Dauod, A.S., El-Qaderi, S.S., et al. (2006) Periodontal Status of Diabetics Compared with Nondiabetics: A Meta-Analysis. Journal of Diabetes and Its Complications, 20, 59-68. https://doi.org/10.1016/j.jdiacomp.2005.05.006

[34] Abiko, Y. and Selimovic, D. (2010) The Mechanism of Protracted Wound Healing on Oral Mucosa in Diabetes. Bosnian Journal of Basic Medical Sciences, 10, 186-191. https://doi.org/10.17305/bjbms.2010.2683

[35] Crano, W.D. and Brewer, M.B. (2002) Chapter 10: Survey Design and Sampling. In: Debra Riegert, Principles and Methods of Social Research, 2nd Edition, Lawrence Erlbaum Associates Publishers, Mahwah, 189-190.

[36] Silness, J. and Löe, H. (1964) Periodontal Disease in Pregnancy. II. Correlation between Oral Hygiene and Periodontal Condition. Acta Odontologica Scandinavia, 22, 121-135. https://doi.org/10.3109/00016356408993968

[37] Löe, H. and Silness, J. (1963) Periodontal Disease in Pregnancy. I. Prevalence and Severity. Acta Odontologica Scandinavia, 21, 533-551. https://doi.org/10.3109/00016356309011240

[38] Zhao, H., Li, C., Lin, L., et al. (2015) Assessment of Alveolar Bone Status in Middle Aged Chinese (40 - 59 Years) with Chronic Periodontitis-Using CBCT. PLoS ONE, 10, e0139553. https://doi.org/10.1371/journal.pone.0139553

[39] Al-Shammari, K.F., Al-Ansari, J.M., Moussa, N.M., Ben-Nakhi, A., Al-Arouj, M. and Wang, H.L. (2006) Association of Periodontal Disease Severity with Diabetes Duration and Diabetic Complications in Patients with Type 1 Diabetes Mellitus. Journal of the International Academy of Periodontology, 8, 109-114.

[40] Abduljabbar, T., Al-Sahaly, F., Al-Kathami, M., Afzal, S. and Vohra, F. (2017) Com- 
parison of Periodontal and Peri-Implant Inflammatory Parameters among Patients with Prediabetes, Type 2 Diabetes Mellitus and Non-Diabetic Controls. Acta Odontologica Scandinavia, 75, 319-324. https://doi.org/10.1080/00016357.2017.1303848

[41] Reza, D., Maryam, T., Bardia, V., et al. (2019) Comparison of the Efficacy of Three Different Instruments in the Removal of Amalgam Overhang: An in Vitro Study. The Journal of Contemporary Dental Practice, 20, 335-340.

https://doi.org/10.5005/jp-journals-10024-2519

[42] Emrich, L.J., Shlossman, M. and Genco, R.J. (1991) Periodontal Disease in Non-InsulinDependent Diabetes Mellitus. Journal of Periodontology, 62, 123-131. https://doi.org/10.1080/00016357.2017.1303848

[43] Taylor, G.W., Burt, B.A., Becker, M.P., et al. (1998) Glycemic Control and Alveolar Bone Loss Progression in Type 2 Diabetes. Annals of Periodontology, 3, 30-39.

https://doi.org/10.1902/annals.1998.3.1.30

[44] Javed, F., Thafeed Alghamdi, A.S., Mikami, T., et al. (2014) Effect of Glycemic Control on Self-Perceived Oral Health, Periodontal Parameters, and Alveolar Bone Loss among Patients with Prediabetes. Journal of Periodontology, 85, 234-241. https://doi.org/10.1902/jop.2013.130008

[45] Wu, Y.Y., Xiao, E. and Graves, D.T. (2105) Diabetes Mellitus Related Bone Metabolism and Periodontal Disease. International Journal of Oral Science, 7, 63-72. https://doi.org/10.1038/ijos.2015.2

[46] Graves, D.T., Oates, T. and Garlet, G.P. (2011) Review of Osteoimmunology and the Host Response in Endodontic and Periodontal Lesions. Journal of Oral Microbiology, 17, 3. https://doi.org/10.3402/jom.v3i0.5304

[47] Jafri, Z., Bhardwaj, A., Sawai, M. and Sultan, N. (2015) Influence of Female Sex Hormones on Periodontium: A Case Series. Journal of Natural Science, Biology, and Medicine, 6, S146-S149. https://doi.org/10.4103/0976-9668.166124

[48] Schulze, A. and Busse, M. (2016) Gender Differences in Periodontal Status and Oral Hygiene of Non-Diabetes and Type 2 Diabetes Patients. The Open Dentisty Journal, 10, 287-297. https://doi.org/10.2174/1874210601610010287

[49] Dahiya, P., Kamal, R. and Gupta, R. (2012) Obesity, Periodontal and General Health: Relationship and Management. Indian Journal of Endocrinology and Metabolism, 16, 88-93. https://doi.org/10.4103/2230-8210.91200

[50] Haber, J., Wattles, J., Crowley, M., et al. (1993) Evidence for Cigarette Smoking as a Major Risk Factor for Periodontitis. Journal of Periodontology, 64, 16-23. https://doi.org/10.1902/jop.1993.64.1.16 